\title{
Neoangiogênese de retalhos cutâneos em ratos tratados com óleo de copaíba
}

\author{
Lígia Reis de Moura Estevão(1), Juliana Pinto de Medeiros(1), Márcia Valéria Rizzo Scognamillo-Szabó(2), \\ Liriane Baratella-Evêncio(3), Ednaldo Carvalho Guimarães ${ }^{(4)}$, Cláudio Augusto Gomes da Câmara(5) \\ e Joaquim Evêncio-Neto ${ }^{(1)}$
}

\begin{abstract}
(1)Universidade Federal Rural de Pernambuco (UFRPE), Departamento de Morfologia e Fisiologia Animal, Rua Manoel de Medeiros, s/no, Dois Irmãos, CEP 52171-900 Recife, PE. E-mail: ligiarme@yahoo.com.br, jupinto2@bol.com.br, evencio@dmfa.ufrpe.br (2)Universidade Estadual Paulista, Faculdade de Medicina Veterinária e Zootecnia, Departamento de Cirurgia e Anestesiologia Veterinária, Distrito de Rubião Júnior, s/non , CEP 18618-000 Botucatu, SP. E-mail: scognamillo_szabo@yahoo.com.br ${ }^{(3)}$ Universidade Federal de Pernambuco, Departamento de Histologia e Embriologia, Rua Moraes Rego, s/no, Cidade Universitária, CEP 50670-901 Recife, PE. E-mail: liriane@uol.com.br ${ }^{(4)}$ Universidade Federal de Uberlândia, Faculdade de Matemática, Av. João Naves de Ávila, no 2121, Campus Santa Mônica, CEP $38400-902$ Uberlândia, MG. E-mail: ecg@ufu.br ${ }^{(5)}$ UFRPE, Departamento de Química. E-mail: camara@dq.ufrpe.br
\end{abstract}

Resumo - $\mathrm{O}$ objetivo deste trabalho foi avaliar morfometricamente a neoangiogênese de retalhos cutâneos subdérmicos em ratos tratados com óleo de copaíba (Copaifera langsdorffii) em pomada a 10\%. O delineamento experimental foi inteiramente casualizado, composto por três recursos farmacológicos. Foram utilizadas dez repetições para cada recurso, e cada animal foi considerado uma unidade experimental. Trinta ratos Wistar foram submetidos à elevação do retalho cutâneo dorsal subdérmico e distribuídos em três grupos: grupo controle absoluto, no qual os animais não receberam nenhum tratamento; grupo controle, no qual os animais receberam tratamento tópico diário com pomada com apenas veículo (glicerina e vaselina); e grupo tratado, no qual os animais foram tratados diariamente com óleo de copaíba em pomada a $10 \%$. Os ratos foram tratados e observados por oito dias após o ato operatório. No oitavo dia de pós-operatório, realizou-se a análise macroscópica do retalho e foram coletados fragmentos das porções cranial, média e caudal do retalho cutâneo para análise histopatológica. A análise morfométrica mostrou diferença significativa para o número de novos vasos sanguíneos nas partes média e caudal do retalho cutâneo no grupo tratado. O óleo de copaíba mostra-se eficiente no aumento da neoangiogênese em retalhos cutâneos subdérmicos de ratos.

Termos para indexação: Copaifera langsdorffii, isquemia, neovascularização.

\section{Neoangiogenesis of skin flaps in rats treated with copaiba oil}

\begin{abstract}
The aim of this work was to evaluate morphometric aspects of subdermic skin flap neoangiogenesis in rats treated with a 10\% copaiba (Copaifera langsdorffii) oil ointment. The experiment design was a completely randomized, with three pharmacological resources. Ten replicates were used for each resource, and each animal was considered one experimental unit. Thirty Wistar rats were submitted to subdermic dorsal skin flap elevation and distributed into three groups: absolute control group, in which the animals did not receive any treatment; control group, in which the animals received topical treatment only with vehicle (glycerin and petroleum jelly), and treated group, in which the animals were treated daily with a $10 \%$ copaiba oil ointment. The rats were treated and observed for eight days after surgery. On the eighth day, the skin flap underwent a macroscopic analysis and fragments were collected from its cranial, medial and caudal portions for histopathological analysis. The morphometric analysis showed significant difference for proliferation of newly formed vessels in the middle and caudal portions of the skin flap in the treated group. Copaiba oil showed efficiency in the increase of neoangiogenesis of subdermic skin flaps in rats.
\end{abstract}

Index terms: Copaifera langsdorffi, ischaemia, neovascularization.

\section{Introdução}

O óleo de copaíba, extraído de árvores do gênero Copaifera, da família Fabaceae-Caesalpinioideae, é uma substância que tem assumido grande importância na medicina natural brasileira (Veiga Junior \& Pinto, 2002). Seus efeitos como substância cicatrizante e anti-inflamatória têm sido pesquisados em vários modelos experimentais.
Brito (1996) estudou os aspectos morfológicos e morfométricos do processo cicatricial de feridas cutâneas abertas em ratos tratadas com óleo de copaíba (Copaifera reticulata) e observou aumento da crosta da lesão, do tecido de granulação e do número de vasos sanguíneos, com diminuição do número de fibras colágenas. O óleo de copaíba (C. langsdorffii) possui a capacidade de reduzir o tempo de reparação tecidual em 
feridas cutâneas em ratos (Eurides et al., 1998; Paiva et al., 2002), reduz a formação de tecido de granulação (Basile et al., 1988), além de possuir propriedades anti-inflamatórias e analgésicas (Fernandes et al., 1992).

Os retalhos cutâneos constituem-se de segmentos de pele obtidos de uma área doadora e transferidos para uma região receptora, mantendo uma conexão temporária ou definitiva por meio de um pedículo, de onde vem o suprimento sanguíneo. Sobrevivem em decorrência de sua circulação intacta, permitem cobertura imediata do leito de um ferimento e evitam cicatrização prolongada, formação de cicatriz excessiva e contratura associada com cicatrização por segunda intenção (Fossum et al., 2002). São versáteis e de fácil execução, apresentam menor custo do manejo da ferida e recobrimento piloso adequado, quando empregados em cães (Sakuma et al., 2003).

A vitalidade dos retalhos de pele e a incorporação dos retalhos cutâneos são fundamentais para o sucesso terapêutico de técnicas reconstrutivas. A evolução positiva depende do suporte vascular do leito receptor, do pedículo vascular dominante do retalho, de alterações locais, como a resposta inflamatória e o processo de cicatrização, e de alterações sistêmicas, ou seja, da resposta metabólica ao trauma (Almeida et al., 2004). São classificados em retalhos randômicos, quando não é considerada a sua vascularização inerente, e axiais, quando incorporam uma artéria e veia cutânea diretas.

O uso de substâncias que estimulam a angiogênese é comumente empregado para melhorar a viabilidade do retalho. Fármacos vasodilatadores, vasoativos, bloqueadores de canais de cálcio e antioxidantes (Biondo-Simões et al., 2000; Almeida et al., 2004; Esteves Junior et al., 2005) foram pesquisados. O uso do fitoterápico Ginkgo Biloba (EGB) foi estudado por Souza Filho et al. (2002). Outras pesquisas avaliaram os efeitos de alguns recursos não farmacológicos, como a estimulação elétrica nervosa transcutânea (Liebano et al., 2003) e o laser de baixa potência (Branco et al., 2005).

Tomando por base os resultados positivos obtidos com o óleo de copaíba em vários modelos de cicatrização somados à necessidade de recursos terapêuticos na melhora da viabilidade do retalho cutâneo, e visando ao estudo de uma manipulação de pomada em concentração suficiente para favorecer o processo de reparação tecidual, este trabalho visa a avaliar morfometricamente a neoangiogênese de retalhos cutâneos subdérmicos em ratos tratados com óleo de copaíba em pomada a $10 \%$.

\section{Material e Métodos}

Foram utilizados 30 ratos Wistar (Rattus norvegicus albino) machos, pesando entre 410 e $430 \mathrm{~g}$, com três meses de idade, provenientes do Biotério do Departamento de Morfologia e Fisiologia Animal (DMFA) da Universidade Federal Rural de Pernambuco (UFRPE). Os ratos foram distribuídos aleatoriamente em três grupos de dez animais cada um e mantidos em caixas individuais, em ambiente com temperatura de 23 a $25^{\circ} \mathrm{C}$ e ciclo claro/escuro controlado de $12 \mathrm{em}$ 12 horas. Receberam ração comercial para roedores e água à vontade.

Este trabalho foi aprovado pelo Comitê de Experimentação e Ética Animal do DMFA da UFRPE. Após a ortotanásia, os animais foram preparados, congelados e descartados segundo as exigências dos princípios éticos para trabalhos experimentais do Colégio Brasileiro de Experimentação Animal (COBEA), São Paulo, Brasil, 1991.

O delineamento utilizado foi o inteiramente casualizado (DIC), composto por três recursos farmacológicos. Foram utilizadas dez repetições para cada recurso, e cada animal foi considerado uma unidade experimental. Os grupos experimentais foram denominados grupo controle absoluto (GCA), que não recebeu tratamento; grupo controle (GC), no qual os animais, após a cirurgia de retalho cutâneo, receberam tratamento tópico com pomada com apenas vaselina e glicerina como veículo; e grupo tratado (GT), no qual os animais receberam tratamento tópico com pomada contendo óleo de copaíba na concentração de $10 \%$.

O óleo de copaíba foi extraído de árvores nativas de C. langsdorffii da região do Alto Paranaíba, Minas Gerais, catalogadas e registradas sob o número HM00217 no Herbário Mandevilla da Faculdade de Ciências Agrárias do Centro Universitário de Patos de Minas. O óleo foi analisado no Departamento de Química da UFPE. A análise cromatográfica (CG) foi obtida utilizando um aparelho Hewlett Packard 5890 Series II equipado com um detector de ionização de chama (FID) e com uma coluna capilar de sílica fundida J \& W Scientific DB-5 (30 m x 0,25 mm x $0,25 \mu \mathrm{m})$. As temperaturas do injetor e do detector 
foram de 220 e $285^{\circ} \mathrm{C}$, respectivamente. Foi usado hélio como gás de arraste, a uma vazão de $1 \mathrm{~mL} \mathrm{~min}^{-1}$. $\mathrm{O}$ programa de temperatura da coluna foi de $40^{\circ} \mathrm{C}$ $(1 \mathrm{~min})$ até $220^{\circ} \mathrm{C}$ a $4^{\circ} \mathrm{C} \min ^{-1} ; 220^{\circ} \mathrm{C}$ até $280^{\circ} \mathrm{C}$ a $20^{\circ} \mathrm{C} \mathrm{min}^{-1}$. Os espectros de massas foram obtidos com um impacto eletrônico de 70 e V, 0,84 scan s-1 de $\mathrm{m} \mathrm{z}^{-1}$ 40 a 550. O hidrogênio foi usado como gás de arraste, com velocidade de fluxo de $1,5 \mathrm{~mL} \mathrm{~min}^{-1}$, no modo split (1:10). Uma solução de $1,5 \mu \mathrm{L}$ com $10 \mathrm{mg}$ de óleo em acetato de etila foi injetada. Os índices de retenção foram obtidos pela injeção do óleo com uma mistura de hidrocarbonetos lineares $\mathrm{C}_{11}-\mathrm{C}_{24}$ e calculados de acordo com a equação de Van den Dool \& Kratz (1963). Os compostos foram identificados com base na comparação dos índices de retenção calculados com aqueles disponíveis na literatura, seguida pela comparação do fragmentograma de padrões das massas (Adams, 1995), bem como pela comparação direta das sugestões das massas disponíveis na biblioteca do computador (Wiley, com 250.000 compostos), contemplando apenas as similaridades entre os fragmentogramas.

A confecção do retalho cutâneo dorsal subdérmico de base cranial, com $8 \mathrm{~cm}$ de comprimento e $3 \mathrm{~cm}$ de largura, foi realizada conforme Acevedo-Bogado et al. (2002) modificado, respeitando como limites anatômicos craniais os ângulos inferiores das escápulas. Os animais foram anestesiados com anestesia dissociativa com xilazina na dose de $3 \mathrm{mg} \mathrm{kg}^{-1} \mathrm{e}$ cetamina a $10 \mathrm{mg} \mathrm{kg}^{-1}$, por via intramuscular, segundo Andrade (2002). Foram posteriormente posicionados em decúbito ventral, com imobilização dos membros ao plano da mesa usando fita adesiva. Após tricotomia e antissepsia com clorexidina tópica alcoólica a $0,5 \%$, $\mathrm{a}$ área foi demarcada usando caneta cirúrgica e moldes previamente confeccionados em papel cartolina e esterilizados. O retalho cutâneo preestabelecido, constituído por fáscia superficial, panículo carnoso, tecido subcutâneo e pele, foi confeccionado utilizando bisturi de lâmina número 15 , dissecado e deslocado do plano músculo-aponeurótico adjacente, com dissecção romba, elevado do leito, reposicionado e ressuturado com sutura simples interrompida utilizando fio monofilamentoso de náilon 4.0 agulhado.

Imediatamente após a execução do retalho, os ratos do GT foram submetidos a tratamento tópico com o óleo de copaíba em pomada a $10 \%$. Esse procedimento foi repetido a cada 24 horas, completando um total de sete curativos, durante os quais todos os animais eram avaliados clinicamente. Os ratos do grupo controle foram tratados topicamente com pomada contendo apenas vaselina e glicerina durante o mesmo período de tempo. Os animais do grupo controle absoluto não receberam tratamento.

Decorridos oito dias de tratamento, os animais foram anestesiados usando isoflurano e fotografados usando câmera digital (Sony W70) a uma distância focal de $20 \mathrm{~cm}$. Essas fotografias foram posteriormente digitalizadas com resolução de 640 pontos na horizontal e 480 pontos na vertical, 24 bits de cores, com demarcação da área de necrose e área total e com posterior cálculo da percentagem da área de necrose.

Após a análise macroscópica, os animais foram ortotanasiados por aprofundamento do plano anestésico e foram coletados três fragmentos do retalho cutâneo dorsal de áreas distintas: cranial, com tecido sadio; média, com tecido de transição, situada entre as áreas cranial e caudal; e caudal, com área de necrose. Os fragmentos foram fixados em líquido de Boüin por seis horas e transferidos para álcool a $70 \%$. Em seguida, foram desidratados em etanol, diafanizados em xilol, processados para inclusão em parafina pelos métodos de rotina, cortados em micrótomo tipo Minot ajustado para $5 \mu \mathrm{m}$ e colocados em lâminas previamente untadas com albumina de Mayer. Após secagem, os cortes foram corados por hematoxilina e eosina (HE). O material foi analisado e fotografado em microscópio de luz Olympus BX-49. Para cada região do retalho cutâneo foram obtidas dez imagens, que foram analisadas em um software analisador de imagens (Imagelab 2000), pelo qual foi possível quantificar o número de vasos sanguíneos neoformados.

Os dados obtidos no oitavo dia pós-operatório para a variável número de vasos sanguíneos foram submetidos à análise de variância, e as médias foram comparadas pelo teste de Tukey, a 5\% de probabilidade. As análises foram feitas usando o software SISVAR (Banzatto \& Kronka, 2006).

\section{Resultados e Discussão}

Neste estudo, o rato foi adotado como modelo de experimentação devido à facilidade na obtenção, manuseio e acomodação, à resistência à manipulação, a agressões cirúrgicas e a processos infecciosos, bem como ao fato de esse animal ser um modelo experimental 
aceito internacionalmente. Não foram observados processos de irritação local, alergias ou possíveis inflamações que fossem provocadas pelo veículo utilizado na formulação.

O retalho cutâneo efetuado foi do tipo randômico, por ser o mais utilizado na medicina veterinária para cães e gatos, segundo Fossum et al. (2002). A determinação do tamanho da área dos retalhos cutâneos randômicos é frequentemente difícil, porque eles apresentam uma nutrição ao acaso, o que facilita uma maior possibilidade de isquemia e necrose. A relação entre a largura do pedículo e o comprimento do retalho cutâneo é fundamental no estabelecimento da sua viabilidade. Neste experimento, o tipo de retalho cutâneo estudado teve relação comprimento-largura suficientemente aumentada para que pudessem ser avaliados os efeitos de um retorno venoso dificultado. Optou-se pela realização de um retalho de $8 \times 3 \mathrm{~cm}$ com proporções de 2,6:1 por ser esse um tamanho intermediário entre aqueles de uso corrente na literatura (Acevedo-Bogado et al., 2002; Almeida et al., 2004; Branco et al., 2005).

O período estabelecido para observação dos retalhos foi até o oitavo dia de pós-operatório. Nessa ocasião, a eventual área de necrose já se encontra definida e o processo de angiogênese e formação de neovascularização a partir do leito e da área perimetral do retalho já estão instalados (Ferreira, 1995).

O óleo de copaíba utilizado apresentou um perfil químico semelhante ao dos óleos de copaíba pesquisados por Veiga Junior \& Pinto (2002), com regiões diterpênicas e sesquiterpênicas características (Tabela 1). Segundo esse autor, a concentração e a natureza dos sesquiterpenos

Tabela 1. Composição química do óleo de copaíba testado.

\begin{tabular}{lcc}
\hline Composto $^{(1)}$ & $\begin{array}{c}\text { Índice de retenção } \\
\text { (IR) obtido }\end{array}$ & $\begin{array}{c}\text { Percentagem } \\
\text { relativa }\end{array}$ \\
\hline$\alpha$-cubebeno (Sno) & 1.359 & 2,37 \\
Ciclosaviteno (Sno) & 1.370 & 1,51 \\
$\alpha$-Copaeno (Sno) & 1.386 & 5,80 \\
$\beta$-cariofileno (Sno) & 1.429 & 2,45 \\
$\gamma$-muuroleno (Sno) & 1.480 & 22,73 \\
$\gamma$-Patchouleno (Sno) & 1.501 & 1,59 \\
Trans- $\beta$-guaieno (Sno) & 1.506 & 4,32 \\
$\alpha$-epóxido de cedreno (So) & 1.575 & 4,95 \\
Zizanona (So) & 1.670 & 4,41 \\
(Z)- $\alpha$-santalol (So) & 1.673 & 1,39 \\
Mustakona (So) & 1.678 & 2,08 \\
Curcumenol (So) & 1.734 & 2,45 \\
Eremofilona (So) & 1.741 & 6,77 \\
Abieta-8,12-dieno (Di) & 2.028 & 3,79 \\
Caureno (Di) & 2.046 & 6,84 \\
9-octadecenoato de metila (DAC) & 2.091 & 26,54 \\
\hline Total & & 99,99 \\
\hline
\end{tabular}

${ }^{(1)}$ Sno, sesquiterpenos não oxigenados; So, sesquiterpenos oxigenados; Di, diterpenos; DAC, derivado do ácido carboxílico. e diterpenos podem variar, mas essas duas classes de produtos naturais devem estar sempre presentes nos óleos de copaíba. Tappin et al. (2004) trabalharam com diversos óleos de copaíba de árvores nativas do Amazonas e identificaram, em óleos provenientes do Baixo Amazonas, uma predominância de sesquiterpenos, além de teor reduzido de diterpenos, composição semelhante à identificada no óleo testado. O diterpeno ácido copálico, encontrado por Veiga Junior et al. (1997) em todos os óleos comerciais estudados, não foi identificado por Gramosa \& Silveira (2005) em estudo com a espécie $C$. langsdorffii, e também não foi identificado neste experimento. $\mathrm{O}$ cromatograma confirma a autenticidade do óleo e sua espécie, uma vez que os constituintes químicos identificados no óleo testado e no óleo de C. langsdorffii coletado no município de Crato, CE (Gramosa \& Silveira, 2005), foram semelhantes.

Após 24 horas, os retalhos apresentaram uma coloração arroxeada em sua porção caudal, porém sem uma demarcação evidente entre as áreas viável e não viável do retalho. Após três dias de tratamento, os retalhos mostravam uma delimitação mais evidente da intensidade da necrose. No oitavo dia, a necrose estava definida, com delimitação entre a área viável e a não viável. Macroscopicamente o GT apresentou um aspecto menos endurecido da área necrótica, com coloração mais amarelada da área delimitada. Durante a coleta das amostras para exames histológicos, os tecidos com necrose superficial do GT apresentavamse mais irrigados no lado dérmico. Esse fato corrobora os resultados de Brito (1996), Eurides et al. (1998) e Paiva et al. (2002), que observaram aumento do tecido de granulação e vasos sanguíneos durante o processo de reparação tecidual de feridas em cicatrização em ratos, e demonstra que o óleo de copaíba é capaz de aumentar a vascularização.

A avaliação neoangiogênica da pomada contendo óleo de copaíba na concentração de $10 \%$ foi realizada pelo estudo morfométrico dos vasos sanguíneos comumente presentes em um processo de reparação tecidual. O GT apresentou maiores médias de número de vasos sanguíneos nas partes média e caudal do retalho, porém, não houve diferença entre as médias na região cranial do retalho (Figura 1, Tabela 2).

$\mathrm{Na}$ parte cranial do retalho cutâneo de todos os grupos, foram observadas características semelhantes, como o epitélio íntegro e anexos conservados, estroma 
conjuntivo com pouco infiltrado inflamatório, presença de fibroblastos e vasos sanguíneos. Nessa região, a base do pedículo apresentava-se próxima, mantendo a irrigação arterial e a drenagem venosa suficiente para manter a viabilidade do retalho. Esses resultados são característicos da fase de fibroplasia da cicatrização, na qual a migração de fibroblastos é sempre acompanhada de proliferação vascular (Modolin \& Bevilacqua, 1985).

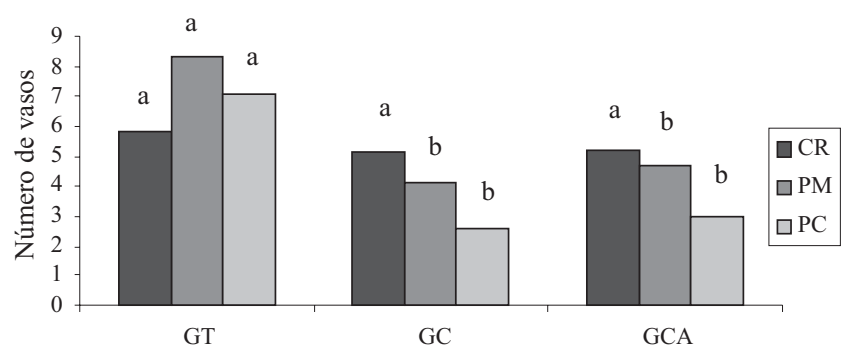

Figura 1. Número de vasos sanguíneos na porção cranial (CR), na porção média (PM) e na porção caudal (PC) nos grupos de animais tratados (GT), controle (GC) e controle absoluto (GCA). Médias com letras iguais, dentro do grupo, não diferem entre si pelo teste de Tukey, a 5\% de probabilidade.

Tabela 2. Média de vasos sanguíneos do retalho cutâneo dorsal de ratos Wistar machos nos grupos tratado (GT), controle $(\mathrm{GC})$ e controle absoluto $(\mathrm{GCA})^{(1)}$.

\begin{tabular}{lccc}
\hline Grupo & Porção cranial & Porção média & Porção caudal \\
\hline GCA & $5,2 \mathrm{aA}$ & $4,7 \mathrm{bA}$ & $3,0 \mathrm{bB}$ \\
GC & $5,1 \mathrm{aA}$ & $4,1 \mathrm{bA}$ & $2,5 \mathrm{bB}$ \\
GT & $5,8 \mathrm{aA}$ & $8,3 \mathrm{aB}$ & $7,1 \mathrm{aAB}$ \\
\hline
\end{tabular}

(1)Médias seguidas de letras iguais, minúsculas na coluna e maiúsculas na linha, não diferem entre si pelo teste de Tukey, a $5 \%$ de probabilidade.
$\mathrm{Na}$ área média do retalho cutâneo, foram observados focos de tecido necrótico e grande infiltrado inflamatório em todos os grupos, porém com uma intensa proliferação de vasos neoformados e maior concentração de fibroblastos na derme e subderme dos retalhos cutâneos do GT. Na parte caudal dos grupos GCA e GC, foram identificadas áreas de necrose tecidual, desestruturação das fibras colágenas e dos anexos da pele e poucos vasos sanguíneos. No GT, esses achados foram similares, porém com maior frequência de vasos sanguíneos neoformados (Figura 2). Nos óleos de copaíba, poucos constituintes ativos identificados foram testados isoladamente, como o beta-bisabolol, que tem ação anti-inflamatória, e o beta-cariofileno, que tem ação bactericida, antitumoral e anti-inflamatória (Veiga Junior et al., 2005). Estudos recentes com ácido caurenoico, obtido do óleo de copaíba de $C$. langsdorffi, demonstraram que esse diterpeno apresenta diferentes propriedades, como ação anti-inflamatória, vasorrelaxante, relaxante do músculo liso e protetora de colite induzida por ácido acético (Veiga Junior et al., 2005). Os resultados aqui descritos permitem inferir que a pomada de óleo de copaíba a $10 \%$ foi responsável por maior angiogênese nas regiões média e caudal do GT, favorecendo o processo de reparação tecidual.

Para a formulação do fitoterápico em pomada, foram utilizados um emoliente (vaselina sólida) e um demulcente (glicerina líquida). Em relação à utilização in natura, as vantagens da pomada incluíram facilidade de aplicação do medicamento sem causar oleosidade excessiva e diminuição da concentração do fitoterápico utilizado. A eficácia também se comprovou ao provocar
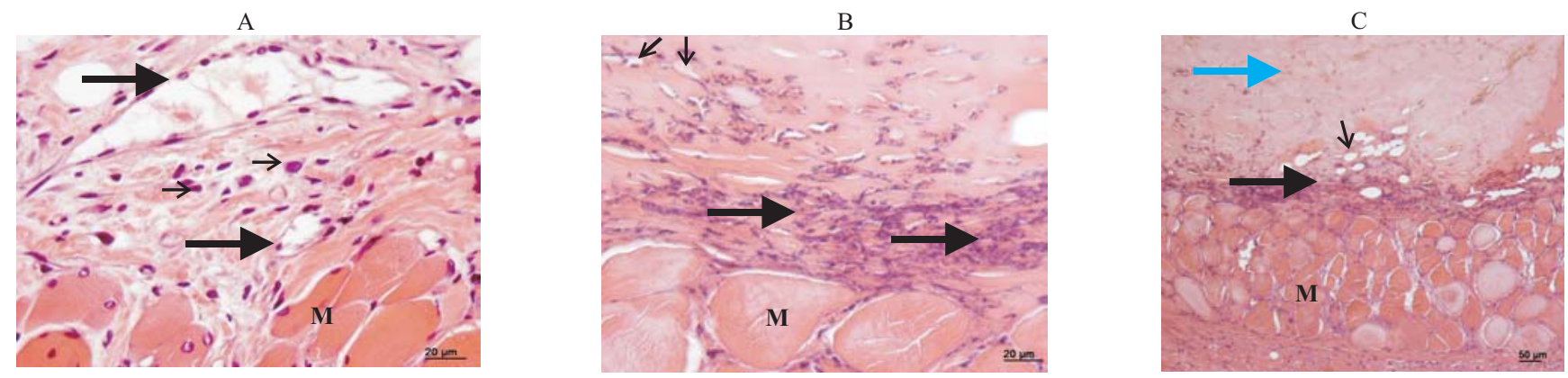

Figura 2. Fotomicrografia do retalho cutâneo da porção caudal de ratos Wistar em coloração HE. A: grupo tratado, com presença de vasos sanguíneos (setas longas), infiltrado leucocitário (setas curtas) e células musculares (M). B: grupo controle, com presença de células em processo de necrose (setas longas), células musculares (M) e poucos vasos (setas curtas). C: grupo controle absoluto, com área necrosada (seta azul), células em processo de necrose (seta longa), células musculares (M) e poucos vasos sanguíneos (seta curta). 
ação hidratante e diminuir a profundidade da perda dos tecidos em decorrência da dessecação das partes isquêmicas mais profundas do retalho, descritas por Nichter et al. (1984).

A contribuição desta pesquisa foi desenvolver uma pomada utilizando óleo de copaíba em concentração suficiente para favorecer o processo de reparação tecidual e ratificar a ação desse óleo na prevenção da necrose, agindo na neoangiogênese. Outras concentrações deverão ser testadas para o aprimoramento da concentração mínima ideal para utilização em retalhos cutâneos.

\section{Conclusão}

A pomada contendo óleo de copaíba de Copaifera langsdorffii na concentração de $10 \%$ aumenta a neoangiogênese do retalho cutâneo subdérmico em ratos.

\section{Agradecimentos}

À Coordenação de Aperfeiçoamento de Pessoal de Nível Superior, pelo suporte financeiro; ao Conselho Nacional de Desenvolvimento Científico e Tecnológico, pela concessão de bolsa de estudos; aos pesquisadores, estagiários e funcionários da Universidade Federal Rural de Pernambuco que participaram da pesquisa direta ou indiretamente.

\section{Referências}

ACEVEDO-BOGADO, C.E.; BINS-ELY, J.; ACAMPORA, A.J. d'; NEVES, R. d'E. Efeito da hialuronidase na sobrevida de retalhos cutâneos em ratas. Acta Cirúrgica Brasileira, v.17, p.14-16, 2002.

ADAMS, R.P. Identification of essential oil components by gas chromatography/mass spectroscopy. Carol Stream: Allured Publishing Corporation, 1995. 468p.

ALMEIDA, K.G. de; FAGUNDES, D.J.; MANNA, M.C.B.; MONTERO, E.F.S. de. Ação do dimetil-sulfóxido na isquemia de retalhos randômicos de pele em ratos. Acta Cirúrgia Brasileira, v.19, p.648-657, 2004.

ANDRADE, S.F. Manual de terapêutica veterinário. São Paulo: Roca. 2002. 697p.

BANZATTO, D.A.; KRONKA, S.N. Experimentação agrícola. Jaboticabal: Funep, 2006. 237p.

BASILE, A.C.; SERTIÉ, J.A.A.; FREITAS, P.C.D.; ZANINI, A.C. Anti-inflammatory activity of oleoresin from Brazilian Copaifera. Journal of Ethnopharmacology, v.22, p.101-109, 1988.
BIONDO-SIMÕES, M. de L.P.; GRECA, F.H.; PAULA, J.B.; CHIN, E.W.K.; SANTOS, E.A.A. dos; STALHSCHMIDT, F.L. Effect of buflomedil on ischemic skin flaps: experimental study in rats. Acta Cirúrgica Brasileira, v.15, p.1-7, 2000.

BRANCO, C.H.; OLIVEIRA, P.R. de; ESTEVES JÚNIOR, I.; LIEBANO, R.E.; BALDAN, C.; GOMES, A.C. Corrente direta na viabilidade de retalho cutâneo randômico em ratos. Revista do Instituto de Ciências da Saúde, v.23, p.11-17, 2005.

BRITO, N.M.B. Aspectos morfológicos e morfométricos da cicatrização de feridas cutâneas abertas em ratos tratados com óleo de copaíba. 1996. 56p. Dissertação (Mestrado) - Escola Paulista de Medicina, São Paulo.

DOOL, H. van D.; KRATZ, P.D. A generalization of the retention index system including linear temperature programmed gas-liquid partition chromatography. Journal of Chromatography, v.11, p.463-471, 1963.

ESTEVES JUNIOR, I.; MASSON, I.B.; FERREIRA, L.M.; LIEBANO, R.E.; BALDAN, C.; GOMES, A.C. Topical administration of hydralazine hydrochloride on the viability of random skin flaps in rats. Acta Cirúrgica Brasileira, v.20, p.164-167, 2005.

EURIDES, D.; MAZZANTI, A.; GONÇALVES, G.F.; BELLETI, M.E.; SILVA, L.A.F.; FIORAVANTI, M.C.S.; CHAVES, N.S.T.; BOMBONATO, P.P.; CAMPOS, V.A.; OGATA, A.S. Aspectos morfológicos, morfométricos e histológicos da reparação tecidual de feridas cutâneas de camundongos tratadas com óleo de copaíba (Copaifera langsdorffii). Veterinária Notícias, v.4, p.77-82, 1998. FERNANDES, R.M.; PEREIRA, N.A.; PAULO, L.G. Anti-inflamatory activity of copaíba balsam (Copaifera cearensi). Revista Brasileira de Farmácia, v.73, p.53-56, 1992.

FERREIRA, L.M. Retalhos cutâneos. In: FERREIRA, L.M. Manual de cirurgia plástica. São Paulo: Atheneu, 1995. p.45-62.

FOSSUM, T.W.; HEDLUND, C.S.; HULSE, D.A.; JOHNSON, A.L.; SEIM, H.B.; WILLARD, M.D.; CARROLL, G.L. Cirurgia do sistema tegumentar. In: FOSSUM, T.W. Cirurgia de pequenos animais. São Paulo: Roca, 2002. p.101-171.

GRAMOSA, M.V.; SILVEIRA, E.R. Volatile constituents of Copaifera langsdorffii from the Brazilian northeast. Journal of Essential Oil Research, v.17, p.130-132, 2005.

LIEBANO, R.E.; FERREIRA, L.M.; SABINO NETO, M. Modelo experimental para estimulação elétrica nervosa transcutânea em retalho cutâneo randômico isquêmico em ratos. Acta Cirúrgica Brasileira, v.18, p.54-59, 2003.

MODOLIN, M.; BEVILACQUA, R.G. Cicatrização das feridas: síntese das aquisições recentes. Revista Brasileira de Clínica e Terapêutica, v.14, p.208-213, 1985.

NICHTER, L.S.; SOBIESKI, M.W.; MORGAN, R.F.; RODEHEAVER, G.; EDLICH, R.F. Quantitation of skin-flap survival: a computer-based method. Plastic and Reconstructive Surgery, v.73, p.684-686, 1984.

PAIVA, L.A.F.; ALENCAR CUNHA, K.M; SANTOS, F.A.; GRAMOSA, N.V.; SILVEIRA, E.R.; RAO, S.N. Investigation on the wound healing activity of oleo-resin from Copaifera langsdorffii in rats. Phytotherapy Research, v.16, p.737-739, 2002. 
SAKUMA, C.H.; MATERA, J.M.; VALENTE, N.S. Estudo clínico sobre aplicação do retalho cutâneo pediculado em cirurgia oncológica no cão. Brazilian Journal of Veterinary Research and Animal Science, v.40, p.32-37, 2003.

SOUZA FILHO, M.V.P. de; LEAL, P.R. de A.; SBALCHIERO, J.C.; MARQUES, M.J. Efeitos do extrato de Ginkgo Biloba na sobrevida de retalhos cutâneos em ratos. Revista da Sociedade Brasileira de Cirurgia Plástica, v.17, p.45-56, 2002.

TAPPIN, M.R.R.; PEREIRA, J.F.G.; LIMA, L.A.; SIANI, A.C. Análise química quantitativa para a padronização do óleo de copaíba por cromatografia em fase gasosa de alta resolução. Química Nova, v.27, p.236-240, 2004.

VEIGA JUNIOR, V.F.; PATITUCCI, M.L.; PINTO, A.C. Controle da autenticidade de óleos de copaíba comerciais por cromatografia gasosa de alta resolução. Química Nova, v.20, p.612-615, 1997.

VEIGA JUNIOR, V.F.; PINTO, A.C. O gênero Copaifera L. Química Nova, v.25, p.273-286, 2002.

VEIGA JUNIOR, V.F.; PINTO, A.C.; MACIEL, M.A. Plantas medicinais: cura segura? Química Nova, v.28, p.519-528, 2005.

Recebido em 3 de dezembro de 2008 e aprovado em 30 de abril de 2009 